\title{
Wavelength dependent birefringence of surface plasmon polaritonic crystals
}

\author{
Jill Elliott, ${ }^{1}$ Igor I. Smolyaninov, ${ }^{2}$ Nikolay I. Zheludev,${ }^{3}$ and Anatoly V. Zayats ${ }^{1, *}$ \\ ${ }^{1}$ School of Mathematics and Physics, The Queen's University of Belfast, Belfast BT7 1NN, United Kingdom \\ ${ }^{2}$ Electrical and Computer Engineering Department, University of Maryland, College Park, Maryland 20742, USA \\ ${ }^{3}$ School of Physics and Astronomy, University of Southampton, Southampton SO17 1BJ, United Kingdom
}

(Received 1 June 2004; published 6 December 2004)

\begin{abstract}
A periodic array of elliptical nanoholes in a metal film acts as a thin two-dimensional birefringent crystal with wavelength dependent orientation of principal polarization eigenstates, the property which is not encountered in natural crystals. The transmission spectrum of such metallic nanostructures can be controlled in a wide range by selecting the polarization of incident and transmitted light.

DOI: 10.1103/PhysRevB.70.233403

PACS number(s): 78.66.Bz, 42.70.Qs, 42.81.Gs, 78.67.-n
\end{abstract}

Optical birefringence and dichroism are well-understood manifestations of the anisotropy of crystalline structures. Among 32 three-dimensional crystal point groups, 27 sustain optical anisotropy: for a given direction of the wave propagation there are two polarization states which are eigenstates of a crystal. ${ }^{1,2}$ The polarization of eigenstates does not change during propagation in a crystal. Disregarding nonlocality, these eigenstates are linear polarizations bound to the main crystallographic directions. They do not depend on the frequency of the electromagnetic wave. If one would monitor the transmission of linearly polarized light through an absorptive crystal or a thin slice of it, losses would reach their extremes for the same polarization state, whatever the light wavelength is.

In this paper we report that a thin metal film with a regular square array of elliptical nanoholes acts alike a thin birefringent crystal but shows a remarkable difference from natural crystals: transmission losses (or enhancement) through the nanostructure reach their extremes for linear polarization states which directions resolutely depend on the wavelength. This is the effect of fundamental importance for optical electrodynamics and might reveal itself in other systems (e.g., photonic crystals or conventional crystals at x-ray wavelengths) when a lattice scale is on the order of the light wavelength and thus conventional crystal optics, developed with the assumption that the wavelength is much larger than the lattice period, cannot be applied. ${ }^{3}$ This unique effect underpins the rich coloring of the structure which is seen in polarized white light. We show that such wavelength dependent orientation of polarization eigenstates of the metallic nanostructure can be explained by the interplay between polarization dependent resonant coupling of the incident radiation to the surface plasmon polariton Bloch modes in various directions of the anisotropic Brillouin zone of the periodic structure, which depends on the orientation of the linear polarization of the incident light with respect to the axes of the array and the main axes of the elliptical holes.

Since light does not penetrate deep into metal, the optical properties of metallic nanostructures are determined by the interaction of photons with collective excitations of the conduction electrons close to a metal surface known as surface plasmons. ${ }^{4}$ Different kinds of localized surface plasmon and surface plasmon polariton (SPP) modes can be considered since the collective electron excitations are sensitive to the topography of the metal surface. Not only the spectrum of the surface plasmon states but also the strength of their coupling to photons is determined by a surface structure.

Interactions between photons and surface plasmons are responsible for many exciting and useful optical phenomena such as surface-enhanced Raman scattering and secondharmonic generation, enhanced light transmission through periodically nanostructured metal films, single-photon and controlled photon tunneling, strong polarization-sensitive diffraction and scattering effects on chiral metallic surfaces, transmission of polarization-entangled photons through a metal film, surface polaritonic crystals, etc. ${ }^{5-14}$ These phenomena are dependent on the properties of surface-plasmonrelated electromagnetic states on smooth or periodically nanostructured metal films and/or individual metallic nanostructures and include the electromagnetic field confinement and enhancement, surface polariton band-gap formation, and tunnelling via surface plasmon states.

The coupling and mutual transformations between photons and surface plasmons are determined by the energy and momentum conservation laws as well by the vectorial properties of the incident electromagnetic field. The polarization state of light controls the excitation of surface polaritons in one or another generally nonequivalent directions of the Brillouin zone of the periodic surface structure. ${ }^{14}$ An appropriate choice of the light polarization not only could provide the optimal intensity transmittance through the structure but also could foster various polarization transformation effects related to re-excitation of surface plasmon modes in the structure and, thus, nonlocality of the nanostructure electromagnetic response. ${ }^{12}$

Our experiments demonstrate the polarization properties of SPP behavior in the periodic two-dimensional (2D) arrays of elliptical nanoholes. The transmission of such structure exhibits a broad spectrum which depends on the polarization of the incident light at normal incidence. This is in striking contrast to the transmission of a similar square array of circular holes where the transmission spectrum has well-defined resonances and does not depend on the polarization of the incident light at normal incidence. ${ }^{14}$ Moreover, in the case of elliptical holes, the transmitted light exhibits wavelength dependent conversion of the linearly polarized incident radiation into elliptically polarized transmitted light. This allows control over the spectral composition of the transmitted light 


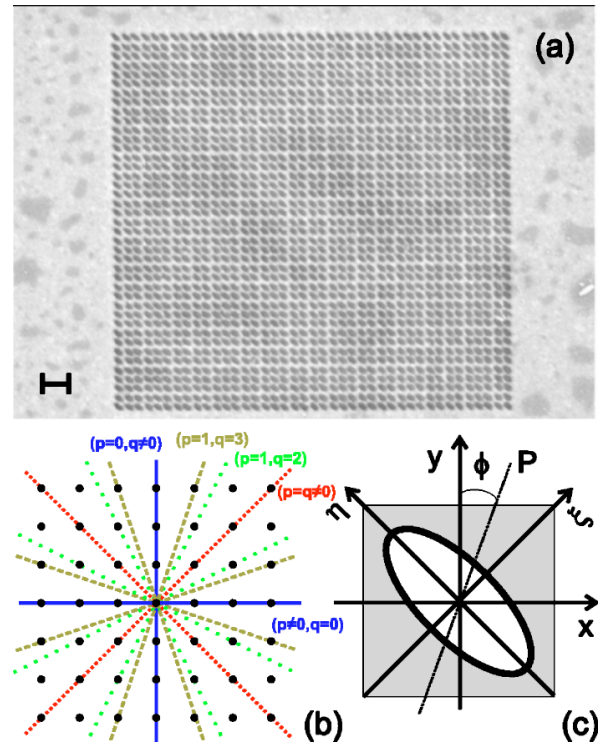

FIG. 1. (a) The image of the periodic array of elliptical holes in a gold film. Scale bar is $2 \mu \mathrm{m}$. (b) (Color on-line) Schematic of a square lattice (point basis) with some of the equivalent directions of SPP excitation corresponding to different directions of the Brillouin zone. (c) A unit cell of the lattice with elliptical basis and related coordinate systems, $\phi$ is the angle between polarization direction of the incident light and the principal $y$ axis of the lattice.

through the polarization state of the incident radiation and by polarization selection of the transmitted light. Such a square array of elliptical holes in a metal film can be considered as a birefringent surface polaritonic crystal.

The metallic nanostructures used in this work were fabricated in a 40-nm gold film on a glass substrate using ion beam milling. The structure consists of elliptical holes with principal axes ratio of about 2, so that the main axes of the ellipse are 250 and $500 \mathrm{~nm}$, respectively (Fig. 1). The holes are arranged in a square array of the periodicity $D=500 \mathrm{~nm}$ so that the main axes of the ellipse are oriented along the bisector of the lattice axes. Thus, a square lattice with an elliptical basis is achieved. The overall size of the structure was $20 \times 20 \mu \mathrm{m}^{2}$. The transmission spectra were recorded using a charge-coupled-device (CCD) spectrograph combined with a long-working-distance optical microscope. Collimated white light from tungsten-halogen source passed through a polarizer was used to illuminate the sample at the normal incidence. The light transmitted through a nanostructure passed through the analyzer was coupled to the fiber bundle connected to a spectrograph. Polarization mode scrambling in the fiber bundle ensures that polarizationrelated effects do not influence spectral measurements.

The normal incidence transmission spectra of the structure obtained without polarization analysis for different polarization of the incident light with respect to the array orientation are shown in Fig. 2. For the polarization azimuth of the incident light parallel to the principal axes of the lattice and, thus, $45^{\circ}$ to the principal axes of ellipses, a continuous "white-light" transmission spectrum is observed which is structureless in the spectral range of $600-700 \mathrm{~nm}$ with a broad peak centered at about $520 \mathrm{~nm}$. If polarization of the

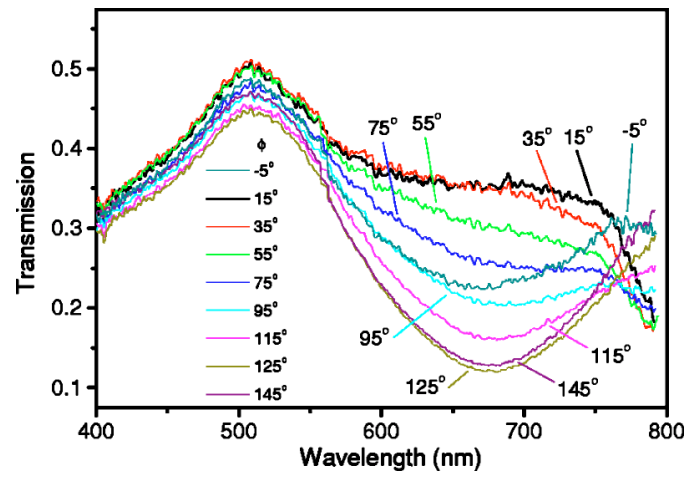

FIG. 2. (Color on-line) Normal incidence transmission spectra of the array of elliptical holes for different polarizations of the incident light without polarization analysis of the transmitted light. Polarization angle $\phi$ is measured with respect to the $y$ axis (Fig. 1).

incident light is rotated relatively to the lattice axes, the transmission spectra exhibit complex, oscillatory behavior different in different spectral ranges (Figs. 2 and 3). For example, for the polarization in the direction of a short principal axis of the ellipses $(\xi)$ the transmission band in the longwavelength spectral range $(750-800 \mathrm{~nm})$ is significantly suppressed while for the polarization along a long principal axis of the ellipses $(\eta)$ the transmission at these wavelengths is enhanced and is suppressed in the 600-700 spectral range. The overall shape of the spectra which depends on the polarization of the incident light suggests that they consist of closely spaced transmission resonances.

The long-wavelength band at around $780 \mathrm{~nm}$ has a transmission minimum for the polarization of light parallel to the short principal axis of ellipses and the maximum for the

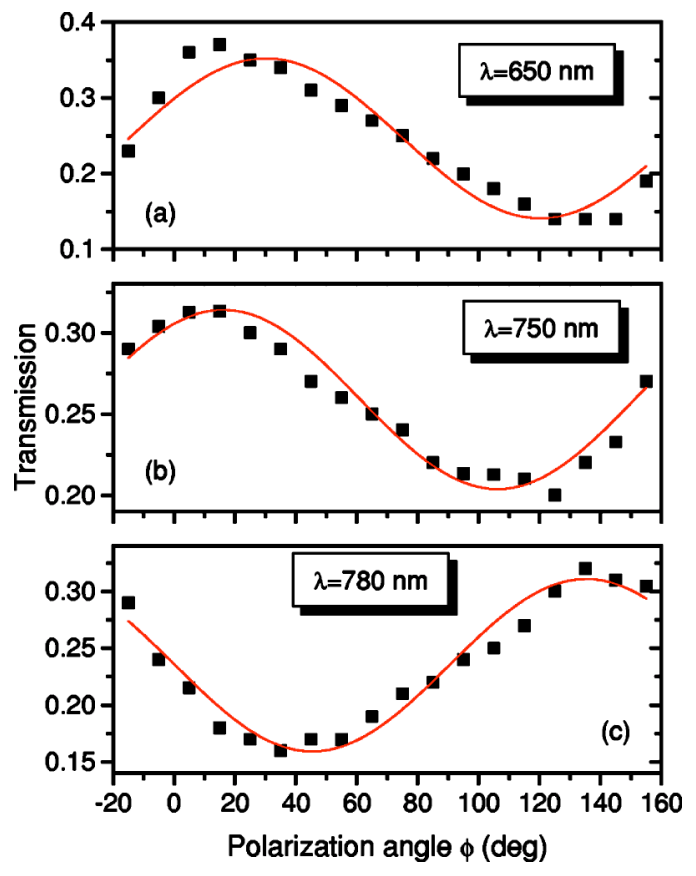

FIG. 3. Polarization dependences of transmission at the wavelengths indicated in the panels plotted from the transmission spectra as in Fig. 2. The polarization angle $\phi$ is measured with respect to the $y$ axis (Fig. 1). 
polarization in perpendicular direction (Fig. 3). For shorter wavelengths, the polarization dependences can be shifted with respect to the dependences observed for the 780-nm transmission by more than $\pi / 4$. All polarization dependences of the transmittance exhibit twofold symmetry of the square array with an elliptical basis. However, in contrast to natural birefringent crystals, the polarization dependences have wavelength-dependent extrema. This spectral polarization effect cannot be explained considering individually polarization properties of a fourfold symmetry square array or twofold symmetry elliptical holes: a square array in a metal film does not provide any polarization dependences of the transmission at normal incidence, while an elliptical hole in a metal film, the same as an elliptical metallic particle, has principal optical axes determined only by its shape. ${ }^{15} \mathrm{Al}$ though polarization conversion effects in diffraction gratings are well known at oblique incidence and the polarization conversion efficiency depends on the wavelength due to various effects including surface plasmon polaritons, ${ }^{8,11}$ wavelength dependent polarization eigenstates have not been observed.

In the case of individual elliptical objects (voids in a metal film or metallic particles) in the electrostatic approximation the polarizability is given by ${ }^{15} \alpha_{i} \sim\left(\varepsilon_{o}-\varepsilon_{s}\right) /\left[\varepsilon_{s}\right.$ $\left.+n_{i}\left(\varepsilon_{o}-\varepsilon_{s}\right)\right]$, where $\varepsilon_{o}$ and $\varepsilon_{s}$ are the dielectric constants of the object and surroundings, respectively, and $n_{i}$ is the socalled depolarization factor depending only on the shape of the object but not the light wavelength. Thus, such an object exhibits anisotropic scattering $E_{i}^{s c} \sim \alpha_{i} E_{i}^{i n}$ with the direction of the polarization eigenstates determined solely by the shape of the object (by $n_{i}$ ) and independent of the wavelength of incident light, $E_{i}^{i n}$. Only the magnitude of $\alpha_{i}$ depends on the wavelength.

To understand the polarizaton dependences of the observed transmission, the spectrum of surface electromagnetic excitations of the array of elliptical holes should be considered. At normal incidence, surface plasmon polaritons can be excited on a metallic nanostructure with a square lattice if the orientation of the electric field vector of the incident light has a component in the direction of SPP propagation: $\left(\mathbf{E} \cdot \mathbf{k}_{S P}\right)$ $\neq 0$. The spectrum of the excited SPP depends on the momentum conservation provided by the diffraction on the nanostructure:

$$
\mathbf{k}_{S P}= \pm p \frac{2 \pi}{D} \mathbf{u}_{x} \pm q \frac{2 \pi}{D} \mathbf{u}_{y},
$$

where $\mathbf{k}_{S P}$ is the wave vector (in the extended Brillouin zone scheme) of the SPP Bloch wave, $\mathbf{u}_{x}$ and $\mathbf{u}_{y}$ are the unit reciprocal lattice vectors of a periodic structure, $D$ is its periodicity assumed to be the same in both $x$ and $y$ directions), and $p$ and $q$ are integer numbers corresponding to the different directions of the SPP Brillouin zone. Thus, for the square lattice (fourfold symmetry) there are two equivalent directions corresponding to $\Gamma-X(p=0, q \neq 0$ and $p \neq 0, q=0)$ and $\Gamma-M(p=q \neq 0)$ directions of the Brillouin zone. However, if the SPP propagates not along the symmetry axes of the lattice, there are four equivalent directions but no eightfold symmetry [Fig. 1(c)].
In the case of an elliptical basis of the lattice, the situation is different. The twofold symmetry of the ellipse reduces overall symmetry properties of the square array. This can be understood taking into account different SPP scattering efficiencies of an ellipse in different (in-plane) directions. In our simple model, this results in the mixing of the Bloch states of the square lattice due an interaction with a basis which has lower symmetry than the symmetry of the square lattice. This will lead to the reduction of the Brillouin zone symmetry from fourfold to twofold symmetry. Therefore, the spectrum of the SPP excitations in the directions, which were degenerated in the lattice with a circular basis, will be split. In this case, there are two symmetry axes related to the $(\xi, \eta)$ axes of the elliptical basis. Thus, by controlling the polarization of the incident light the transmission spectrum can be controlled by excitation or suppression of SPP modes corresponding to different directions of the Brillouin zone. The removed degeneracy of some of the SPP excitations and mixing of the SPP excitations via interaction with the basis result in the variety of closely spaced resonances and observed quasicontinuous transmission spectrum.

In a strict physical consideration, the resonances of the system cannot be treated separately related to a basis and a lattice. Precise treatment of such a system would require the introduction of either Wanier functions instead of Bloch modes or a linear combination of the basis excitations [analogous to the linear combination of atomic orbitals (LCAO's) used in solid-state physics]. ${ }^{16}$ These approaches allow an exact treatment of the symmetry properties of a lattice basis. However, we can understand the wavelength dependent anisotropy of the structure in a simple model. Intuitively, we can consider two types of resonances in the structure related to the lattice and the elliptical basis itself, which has two resonances in the direction of the short and long axes of the ellipse. The competition between these resonances, different at different wavelengths corresponding to different directions of the lattice, results in the observed phenomenon.

It is interesting to compare the polarization properties of an elliptical hole array in a metal film, which forms a SPP crystal, and an array of metallic elliptical particles. Since the electromagnetic interaction between the elements of the array provided by surface polaritons having their own specific polarization properties is needed to achieve wavelength dependent birefringence, in the case of metallic particles deposited on a glass substrate, this effect is not observed. ${ }^{17} \mathrm{Nev}-$ ertheless, one can expect that in the case of an elliptical particle array on a metal surface such effect may exist in the certain frequency range since this system is another realization of a surface polaritonic crystal with an anisotropic basis where polarization dependent coupling between localized surface plasmons and SPPs can be achieved.

Polarization analysis of the transmission allows us to reveal a set of resonances in the broadband transmission spectra. Elliptization of the transmitted light state is observed related to the coupling between resonant excitations in different directions of the lattice of twofold symmetry. The polarized spectra recorded for the light transmitted without polarization changes resemble unpolarized transmission spectra (Fig. 4). Several resonances can be identified by the different 


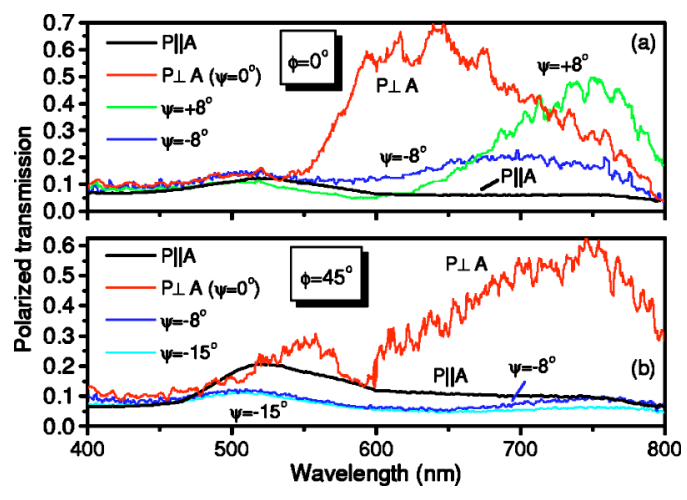

FIG. 4. (Color on-line) Spectra of polarized transmission of the array measured for the incident light polarization (a) $\phi=0^{\circ}$ and (b) $45^{\circ}$. The spectra of the light transmitted without polarization state change $(P \| A)$, with orthogonal polarization $(P \perp A, \psi=0)$, and close to orthogonal polarization $\psi= \pm 8$ and 15 are shown.

degrees of elliptization at around 750, 650, and $550 \mathrm{~nm}$ with other possible unresolved resonances present. At the same time the long-wavelength resonance at around $800 \mathrm{~nm}$ (Fig. 2) related to the symmetry axis of the lattice exhibits a very small degree of elliptization. The elliptization in the transmission depends crucially on the polarization of the incident light. At incident light polarization in the direction of the symmetry axis of the lattice ( $\xi$ or $\eta$ ) the spectral changes of the depolarized light are symmetrical with rotation of the analyzer with respect to the crossed orientation [Fig. 4(b)]. While for the incident polarization $\phi=0^{\circ}$ along the array axis, the similar analyzer rotation results in the nonsymmetrical transmission changes with respect to the clockwise and anticlockwise directions. Thus, by controlling the polarization of the incident and transmitted light, the broadband spectrum of the transmission of the elliptical hole array can be tuned at a specific resonant band corresponding to one or another SPP Bloch mode of the system. This allows us to tune the dominating wavelength of light transmitted through the studied nanostructure in a wide spectral range.

In conclusion, we have studied the polarization properties of the transmission spectra of a periodic array of elliptical holes in a metal film. In contrast to birefringence in natural crystals for which the symmetry of the crystal lattice uniquely determines the polarization eigenstates, the wavelength dependent orientation of the polarization eigenstates in such a metallic structure was observed. This unusual effect shows that conclusions of conventional crystal optics, which are reached in the assumption of the light wavelength being much greater than the lattice period, should be reconsidered for systems for which the wavelength of light is comparable to the lattice period. Reducing the symmetry of the lattice, elliptical holes allow us to achieve an enhanced broadband transmission through the structure, while the transmission spectrum can be controlled by choosing polarizations of the incident and/or transmitted light. This effect can find numerous applications in optical communications and computing where polarization spectral effects can be important for multiplxing and demultiplexing devices. Combined with nonlinear effects which are significantly enhanced in metallic structures due to surface plasmons, the observed polarization properties will allow us achieve active optical control for applications in integrated photonic circuits. Polarization effects are also important for studies of quantum mechanical processes involving many-body electron systems such as surface plasmon polaritons in metallic nanostructures.

We are indebted to C. Cassidi for the sputtering thin gold films used in these experiments and W. Dickson for help with the FIB programs. This work was supported, in part, by EPSRC.
*Corresponding author. Electronic address: a.zayats@qub.ac.uk.

${ }^{1}$ J.F. Nye, Physical Properties of Crystals (Clarendon Press, Oxford, 1976).

${ }^{2}$ S.V. Popov, Y.P. Svirko, and N.I. Zheludev, Susceptibility Tensors for Nonlinear Optics (IOP, Bristol, 1995).

${ }^{3}$ For example, optical trirefringence has been observed in photonic crystals: M.C. Netti, A. Harris, J.J. Baumberg, D.M. Whittaker, M.B.D. Charlton, M.E. Zoorob, and G.J. Parker, Phys. Rev. Lett. 86, 1526 (2001).

${ }^{4}$ H. Raether, Surface Plasmons (Springer, Berlin, 1988).

${ }^{5}$ Surface Polaritons, edited by V.M. Agranovich and D.L. Mills (North-Holland, Amsterdam, 1982).

${ }^{6}$ W.L. Barnes, A. Dereux, and T.W. Ebbesen, Nature (London) 424, 824 (2003).

${ }^{7}$ A.V. Zayats and I.I. Smolyaninov, J. Opt. A: Pure Appl. Opt. 5, S16 (2003).

${ }^{8}$ I.R. Hooper and J.R. Sambles, Opt. Lett. 27, 2152 (2002).

${ }^{9}$ I.I. Smolyaninov, A.V. Zayats, A. Gungor, and C.C. Davis, Phys.
Rev. Lett. 88, 187402 (2002).

${ }^{10}$ I.I. Smolyaninov, A.V. Zayats, A. Stanishevsky, and C.C. Davis, Phys. Rev. B 66, 205414 (2002).

${ }^{11}$ A. Papakostas, A. Potts, D.M. Bagnall, S.L. Prosvirnin, H.J. Coles, and N.I. Zheludev, Phys. Rev. Lett. 90, 107404 (2003).

${ }^{12}$ A.S. Schwanecke, A. Krasavin, D.M. Bagnall, A. Potts, A.V. Zayats, and N.I. Zheludev, Phys. Rev. Lett. 91, 247404 (2003).

${ }^{13}$ E. Altewischer, M.P. van Exter, and J.P. Woerdman, Nature (London) 418, 304 (2002).

${ }^{14}$ L. Salomon, F. Grillot, A.V. Zayats, and F. de Fornel, Phys. Rev. Lett. 86, 1110 (2001).

${ }^{15}$ L.D. Landau and E.M. Lifshits, Electrodynamics of Continuous Media (Pergamon, London, 1984).

${ }^{16}$ C. Kittel, Introduction to Solid State Physics (Wiley, Chichester, 1996).

${ }^{17}$ W. Gotschy, K. Vonmetz, A. Leitner, and F.R. Aussenegg, Opt. Lett. 21, 1099 (1996). 